\title{
LA RESTITUCIÓN: UN MÉTODO LINGÜÍSTICO RECONSTRUCTIVO SINCRÓNICO ${ }^{1}$
}

\author{
Adolfo Constenla Umaña
}

\begin{abstract}
RESUMEN
En este artículo, el autor caracteriza detalladamente la restitución, un método lingüístico reconstructivo sincrónico, destinado a recuperar en lo posible la estructura de lenguas o estados de lengua de los que sólo han quedado datos imperfectamente transcritos desde un punto de vista fonemático.
\end{abstract}

\begin{abstract}
In this article, the author makes a careful characterization of the restitution method. Restitution is a linguistic reconstructive synchronic method made in order to recover languages' structures or languages' states that have only been preserved in imperfect transcriptions.
\end{abstract}

\section{Introducción}

\subsection{Los métodos reconstructivos de la lingüística diacrónica}

Cualquier manual de lingüística diacrónica que se consulte nos ofrecerá dos métodos por medio de los cuales podemos intentar remontarnos en el pasado y reconstruir elementos lingüísticos que ya no podemos observar directamente. Estos métodos, como es bien conocido, son el comparativo y el de reconstrucción interna. Igualmente, en cualquiera de estas exposiciones se nos hará patente el tipo de datos a los que podemos aplicar uno y otro método.

En el caso del método comparativo, enfrentados a datos de distintas lenguas, realizaremos, en primer lugar, en caso de que esto resulte necesario, un diagnóstico por medio del cual determinamos si las lenguas en cuestión presentan relación de parentesco. Esta aplicación diagnosticadora se basa en un supuesto básico de orden metodológico: el reconocimiento de la existencia de un tipo fundamental de cambio, el fonológico, definido como aquel que altera la forma fonológica de las entidades (gramaticales o léxicas) de un mismo modo, en tanto las condiciones de entorno fonético sean idénticas. Cualquier cambio de la forma fonológica que no funcione de este modo no es, por definición, un cambio fonológico. La naturaleza del cambio fonológico hace que, aunque un fonema de la protolengua haya dado distintos resultados en las lenguas descendientes, su existencia previa pueda reconocerse por medio de la correspondencia sistemática entre los fonemas que sean su continuación en ellas.

Una segunda función es precisamente la reconstructiva. Dejando de lado el factor accidental de la cantidad y calidad de los datos disponibles, según el grado de separación de 
las lenguas comparadas, tanto mayor o menor será el número de correspondencias encontradas y la posibilidad de determinar su distribución, factor este último decisivo para poder proceder al aspecto más básico de la reconstrucción de un antepasado común o protolengua: la de su sistema fonemático, sobre la que descansa necesariamente la de cualquier otro nivel de la lengua.

Finalmente, la tercera función del método comparativo es la de subagrupar las lenguas que, a partir del diagnóstico, se han agrupado. Cuando se ha demostrado que varias lenguas están emparentadas, debe determinarse el grado en que lo están, que no necesariamente es el mismo en todos los casos. Si para la agrupación de lenguas lo importante es lo heredado en común, para la subagrupación lo decisivo es más bien lo innovado en común. Las lenguas de una agrupación que comparten de manera exclusiva innovaciones significativas (fonológicas, gramaticales o léxicas) y no atribuibles, por ejemplo, a deriva o a presiones areales, se consideran descendientes de un subantepasado común (una protolengua descendiente a su vez de la protolengua común a la agrupación total).

De las tres aplicaciones, la que aquí nos atañe es la reconstructiva y en lo relativo a la naturaleza de los datos a los que recurre, tenemos que se trata de conjuntos de cognados, esto es, de elementos de las distintas lenguas emparentadas, relacionados por el hecho de ser el resultado de la evolución fonológica de un mismo elemento de la protolengua, un mismo étimo, en cada una de ellas.

El método de reconstrucción interna es menos complejo: se da una única aplicación, como lo indica su nombre: la reconstructiva. Por lo que respecta a los datos por medio de los cuales se da la aplicación a esta finalidad, se trata de morfos de una misma lengua que son cognados o descendientes de un mismo étimo, en términos sincrónicos, alomorfos no supletivos. Las correspondencias, en este caso, se dan entre los fonemas que alternan en los alomorfos, como, por ejemplo, /s/ y /g/ en castellano en formas del verbo decir como /'dise/-/'diga/ o del verbo hacer como /'ase/-/aga/. El dominio de este método es, pues, el de los cambios fonológicos de los que se conserven indicios en alternancias morfofonemáticas que, por otra parte, deben ser recurrentes, no únicas.

Entre las reconstrucciones obtenidas por uno y otro método, como ha señalado Anttila (1972: 274 y siguientes), se da una importante diferencia desde el punto de vista de la profundidad temporal. En el caso del método de reconstrucción interna, ésta es indeterminada, en tanto que en el del método comparativo, es homogénea. La reconstrucción interna produce preformas que pueden datar de cualquier momento anterior al punto de referencia, mientras el método comparativo produce protoformas que se remontan a un punto de separación concreto, un nodo del árbol genealógico. En este uso, el prefijo pre- se refiere a cualquier momento anterior a un nodo y proto- a un momento determinado anterior a un nodo, esto es, a un nodo previo. De acuerdo con lo anterior, el resultado global de la reconstrucción interna es una prelengua.

En suma, los dos métodos comentados obedecen a las siguientes situaciones, por lo que atañe a su naturaleza reconstructiva:

1. Si tenemos datos de varias lenguas, emparentadas de acuerdo con el diagnóstico hecho por el método comparativo, podemos aplicar este mismo método para reconstruir una protolengua.

2. Si tenemos datos de alternancias morfofonemáticas no supletivas y recurrentes, podemos aplicar el método de reconstrucción interna para reconstruir una prelengua. 
En uno y otro caso se trata de recuperar, en la medida en que los datos lo hagan posible, una entidad lingüística que está contenida parcialmente en ellos, pero mezclada con los efectos del cambio, separar lo heredado inicialmente de lo innovado posteriormente. Considero que esta tarea es la característica de los métodos reconstructivos.

\subsection{Necesidad de otro método reconstructivo}

Las situaciones a las que acabo de hacer referencia no son las únicas que hacen necesario algún tipo de reconstrucción. Con frecuencia tenemos datos de una lengua extinta o de un estado anterior de una lengua viva que, como tales, no pueden aprovecharse debidamente para los diversos propósitos para los cuales podemos usar los datos de cualquier lengua. Me refiero al caso, bien abundante en América, de datos de lenguas de sociedades ágrafas transcritos por medio de alguna versión del alfabeto latino (a) con anterioridad al desarrollo en el siglo XX de la lingüística sincrónica a partir del estructuralismo o (b) durante dicho siglo, pero al margen de ella. El caso típico es el de los datos resultantes de lo que Gleason (1961: 349350) ha caracterizado con la expresión "análisis pobres" de la fonología de la lengua y que sintomáticamente se caracterizan por rasgos como los siguientes: enunciación de la pronunciación por referencia imprecisa al valor de las letras en otros idiomas ("las vocales son como las del español"), incapacidad para caracterizar determinadas articulaciones y descarte de rasgos bajo el pretexto de su difícil distinción o presunta baja frecuencia. Ocasionalmente, sin embargo, pueden tratarse de las transcripciones preliminares de un lingüista bien entrenado que pudo recoger algunos datos en un encuentro muy breve con hablantes o semihablantes de la lengua. Estos datos -transcripciones no profesionales o muy preliminares, que denominaré transcripciones asistemáticas-, como aquellos a los que aplicamos los métodos comparativo y de reconstrucción interna, incluyen, por lo menos parcialmente, los elementos lingüísticos que desearíamos poder observar, pero sometidos a distorsiones o alteraciones (cambios, al fin de cuentas) introducidos, no por la evolución lingüística, sino por las limitaciones de los transcriptores que, por lo general, no fueron hablantes nativos.

Aunque exista cierto número de trabajos que pueden considerarse muestras más o menos adecuadas de este tipo de reconstrucción (e.g. Rosenblat 1936, Najlis 1966, Del Río Urrutia 1985, Bertoglia Richards 1988, Mattéi-Muller y Henley 1990, Cerrón Palomino 1995), no conozco ninguna obra que se ocupe explícitamente de su caracterización ni que la plantee como un método en particular, si se exceptúan algunas exposiciones muy breves al respecto contenidas en tesis presentadas en la Universidad de Costa Rica desde 1992 (por ejemplo, Arrieta Espinoza 1992: 12-14, Coronas Urzúa 1993: 6-8).

Es cierto que en los diversos manuales se suele encontrar un capítulo dedicado al uso de los testimonios escritos y a la relación de la disciplina con la escritura, pero normalmente en ellos se tienen en mente las influencias de la escritura sobre el lenguaje (hechos como las pronunciaciones de deletreo, las abreviaturas y los acrónimos, por ejemplo), la preparación por los filólogos de textos en lenguas extintas y temas que se pueden situar dentro de la gramatología (de acuerdo con el sentido dado a este término por Gelb, 1952: 23) y sus diversas manifestaciones, subdisciplinas o aplicaciones como el descifre de escrituras desconocidas, la epigrafía, la paleografía y la grafemática.

Por lo que respecta a lo gramatológico, en los manuales aludidos se trata de problemas planteados por la interpretación de sistemas de escritura u ortografías desarrollados o 
adaptados por los hablantes mismos de las lenguas, consolidados, convencionales y tradicionales empleados por largos períodos de tiempo, y el meollo de la cuestión es el sistema de escritura en sí y su relación convencional con los elementos lingüísticos que representa. En el caso que nos ocupa, aunque los problemas relativos a la escritura jueguen un papel importante (y por ello en las secciones de uso de los testimonios escritos podemos encontrar observaciones aprovechables) es únicamente uno de los elementos que hay que tomar en cuenta y, por lo general, de menor trascendencia que las distorsiones introducidas por los transcriptores.

En cuanto a la filología, se suele destacar su importancia por aportar ediciones críticas de textos que sirven como fuentes fundamentales de datos para el método comparativo. Por ejemplo², Anttila (1972: 43) apunta3: "Antes de que cualquier material escrito pueda usarse para propósitos lingüísticos, el tamiz filológico debe establecer su adecuación a la lengua real, lo mismo que su adecuación a la situación cultural real, si se necesita material semántico." De manera semejante, Jeffers y Lehiste (1979:162) afirman4: "El análisis, interpretación y restauración de textos arcaicos se denomina filología. La investigación filológica sólida es el fundamento de la mayor parte de la investigación en la lingüística histórica" y posteriormente añaden (ibídem: 166) texto dado por lo que respecta a su representación del lenguaje."

A partir del papel de las ediciones críticas de textos de lenguas antiguas de Europa y Asia como fuentes de materiales para el método comparativo (sobre todo en la reconstrucción del protoindoeuropeo y el protosemítico), en el caso de algunos autores estadounidenses, se ha dado en emplear el término filología para hacer referencia a cualesquier análisis lingüísticos de fuentes escritas, incluidos los que podrían considerarse como muestras del tipo de reconstrucción sobre el que versa esta exposición. Como ejemplo de este uso citaré algunos pasajes del conocido mesoamericanista Lyle Campbell: "Las siguientes lenguas se piensa que están extintas... Requieren mucho más trabajo... Se necesitan estudios filológicos de toda la información disponible, incluyendo el estudio de los topónimos, de la onomástica, de los vocabularios, gramáticas y textos que han sobrevivido, etc." (Campbell 1979: 909)6. "La necesidad más importante en el caso del lenca es una investigación filológica concienzuda de las dos lenguas, especialmente del lenca hondureño" (ibídem: 940)7.

En suma, se hace necesaria una exposición que caracterice, por lo menos en sus aspectos fundamentales, este otro método reconstructivo que he propuesto denominar restitución ${ }^{8}$, para contribuir así a su reconocimiento y echar las bases de su desarrollo y refinamiento.

\section{Preliminares del método restitutivo}

Los preliminares del método restitutivo son de dos tipos: la elaboración de un léxico y las cuestiones grafemáticas.

\subsection{Elaboración de un léxico general}

Para poder comparar las distintas transcripciones tanto para efectos de interpretación de los valores fonéticos de las letras como, posteriormente, del establecimiento de correspondencias, 
es necesario reunir todos los materiales léxicos disponibles de la lengua en la forma de un léxico organizado a partir de la lengua en la que se realiza el estudio (por ejemplo, el castellano). Parte muy importante de este trabajo es el reunir bajo un mismo lema todas las versiones de un mismo elemento que las fuentes recogieron bajo distintas traducciones. Por ejemplo, en los materiales del dorasque, lengua extinta del oeste de Panamá, encontramos las siguientes versiones de un mismo lexema que deberían agruparse bajo el lema 'hierba': <baika $>9,<$ baig $>$ 'paja', <bagué> 'sabana', <bagué>, <vagué> 'hierba, paja', <baiké> 'techo', <bágue> 'prado', $<$ baigá> 'yerba'. Como lema se escogerá el significado más general o que se pueda concebir como más básico.

\subsection{Cuestiones grafemáticas}

Aunque lo gramatológico, según se apuntó anteriormente, no sea lo medular en la restitución, sin lugar a dudas es su punto de partida. En este campo, la preocupación principal es de orden eminentemente grafemático, como señaló Hoenigswald (1960: 4), pues el interés es determinar en lo posible el valor fonético (y, en última instancia, fonemático) de los caracteres empleados. El producto final de los preliminares grafemáticos será un inventario de las letras empleadas por cada transcriptor con la determinación de sus valores fonéticos.

En los apartes siguientes se tratan algunos de los aspectos fundamentales que hay que tomar en cuenta para la adecuada determinación de los valores fonéticos de las letras.

\subsubsection{Sistema de escritura empleado}

Se debe determinar ante todo el tipo de sistema de escritura empleado. En la mayor parte de los casos, al menos en América, este será una adaptación del alfabeto de una lengua europea de las más difundidas, particularmente la oficial del país donde se habla la lengua que se desea restituir, un alfabeto fonético, o una mezcla en que, aunque se sigan sobre todo las convenciones alfabéticas de la lengua oficial, se integran algunas procedentes del alfabeto de otra lengua (la materna o la segunda lengua favorita del transcriptor), de un alfabeto fonético o, incluso, enteramente particulares. Así, en el caso de los datos de la lengua cacaopera del Salvador (Bertoglia Richards 1988: 7-8), de las distintas fuentes, tres utilizan el alfabeto castellano con alguna modificación pequeña como el uso de $<\mathrm{k}>$ más apóstrofo para la oclusiva velar glotalizada o de $<\mathrm{h}>$ para la fricativa laríngea sorda y, la cuarta, una versión modificada del alfabeto general de Lepsius (1855). En el del chorotega de Nicaragua, se emplea por lo general el alfabeto castellano, pero uno de los transcriptores (Squier 1860) recurre a los dígrafos $<$ ee> y <oo > para las vocales [i] y [u], otro (Brinton 1886) al dígrafo <ny $>$ para [j] y otro (Lehmann 1920) marca los elementos suprasegmentales por medio de símbolos del alfabeto general de Lepsius.

Con frecuencia, como señaló Hoenigswald (1960: 8-10), la adaptación de un alfabeto conlleva el arrastre a la nueva lengua de inconsistencias entre los grafemas y los fonemas: para algunos efectos, el alfabeto presentará más grafemas de la cuenta; para otros, menos. Por ejemplo, en el caso de adaptaciones del alfabeto castellano, con frecuencia las equivalencias de pronunciación que se dan en las hablas yeístas y seseantes entre $<\mathrm{ll}>\mathrm{y}<\mathrm{y}>$ por una parte, $\mathrm{y}<\mathrm{s}>,<\mathrm{c}>\mathrm{y}<\mathrm{z}>$ por otra, se trasladan a la lengua transcrita. 


\subsection{2. Época en que se recogieron los materiales}

El factor tiempo se debe tomar en cuenta, debido a los cambios de valor de los signos ortográficos según la época. Por ejemplo, en documentos que empleen el alfabeto castellano, $<\mathrm{x}>$ representará con seguridad a [j] en el siglo XVI, mientras que en el siglo XVIII podría estar representando a $[\mathrm{x}]$. Cuando los materiales son anteriores al siglo XIX, la paleografía (si estamos de acuerdo en no limitar el uso del término al estudio de las grafías medievales) resulta de mucha utilidad. Por ejemplo, en escritos de hasta el siglo XVIII, es posible encontrar el uso de la misma letra, $<\mathrm{f}>$ para representar tanto [s] como [f].

\subsubsection{Las tradiciones locales}

Aun en una misma época, pueden darse distintas tradiciones locales que hay que tomar en cuenta. Por ejemplo, en Guatemala el valor de $<x>$ como [j]] en la representación de lenguas indígenas arraigó de tal modo que se ha mantenido, prácticamente sin excepción, hasta nuestros días, a pesar de los cambios ocurridos al respecto en la ortografía castellana. En la Gramática del idioma cachiquel de Rosales (escrita en 1748), aunque no se incluye entre las letras de pronunciación diferente sino entre las que "se pronuncian de la misma manera que las pronunciamos en Castilla", es obvio que $<x>$ representaba la fricativa palatoalveolar, no la velar. En cambio, en Colombia, si bien en el siglo XVII todavía se encuentra en los materiales muiscas con el valor de [j] (Constenla Umaña 1984: 85-86), en el siglo XVIII, en el léxico andaquí enviado a España en 1787 por José Celestino Mutis, se usa exclusivamente con el mismo valor que $<\mathrm{j}>$ ([h], de acuerdo con Coronas Urzúa 1993: 44).

\subsubsection{Los rasgos personales en la grafía}

En la medida de lo posible, sería conveniente poder consultar los manuscritos originales autógrafos, pues alguna particularidad de la grafía del transcriptor puede haber sido malinterpretada por quienes hayan trasladado sus datos a la imprenta. Por ejemplo, la $<\mathrm{c}>$ de algunas personas puede ser confundida con $<\mathrm{e}>$. Esto parece haber ocurrido en el caso de la palabra jirajara que significa 'paraulata' (un tipo de ave) que, en una misma fuente (Oramas 1916), aparece transcrita de las siguientes dos maneras $<$ kokrí $>$ y $<$ koerí $>$.

\subsubsection{Las observaciones hechas por los transcriptores y cualquier otro observador}

Aunque con frecuencia sean de difícil interpretación, las observaciones hechas por los transcriptores y, en general, por cualquiera que haya observado la lengua, pueden resultar de gran utilidad para establecer las equivalencias fonéticas de las letras usadas. Por ejemplo, del valor del dígrafo <ch > en muisca, la única aclaración con que contamos es la siguiente del Diccionario y gramática chibcha, obra anónima del siglo XVII (González de Pérez 1987: 71): "La $2^{\mathrm{a}} \mathrm{p}$ [ro]nunçiaçión es destas sylabas, cha, che, chi, cho, chu, la qual pronunçiaçión no se a de hazer con toda la lengua sino con la punta no más." Esta observación basta, sin embargo, para dejar bien en claro que el sonido representado no pudo ser una predorsal como [ț̣] o [j]], como han planteado varios autores (véase Constenla Umaña 1984: 75-79).

\subsubsection{Los préstamos}

La adaptación de los préstamos tomados por otras lenguas de la lengua analizada o viceversa, en tanto la fonología de dichas otras lenguas nos sea bien conocida, puede constituir 
un importante indicio. Por ejemplo, en el caso del muisca, gallina se adoptó como caína en tanto daga se adoptó como raga . Esto sugiere que el fonema muisca representado por medio de $<$ g $>$ era fricativo o aproximante como la realización del fonema /g/ castellano en posición intervocálica, no oclusivo, como la realización de este fonema en posición inicial absoluta.

\subsubsection{La comparación de transcripciones}

Cuando una letra puede representar dos o más valores fonéticos como ocurre hasta el siglo XVIII en el alfabeto castellano con $<\mathrm{f}>$ (que puede representar tanto una fricativa labial como una alveolar) o $<\mathrm{v}>$ (que puede representar tanto una vocal posterior alta, como una semiconsonante labiovelar o una consonante labial, fricativa u oclusiva), uno de los recursos que nos permiten conocer el valor que le da un transcriptor determinado, tanto en ejemplos concretos como en general, es la comparación de sus transcripciones con las de otro. Por ejemplo, en el caso del muisca, el valor de $<\mathrm{v}>$ en la transcripción vaya 'madre' que aparece en la gramática de Bernardo de Lugo (1619) se aclara al compararla con la transcripción de la misma palabra en la obra anónima del mismo siglo Diccionario y gramática chibcha (González de Pérez 1987): guaya.

\subsubsection{Los resultados del método comparativo y el conocimiento tipológico areal y de lin-} güística general como elementos auxiliares en el análisis grafemático

En ocasiones, o no hay aclaraciones respecto del valor fonético de una letra o estas son claramente insuficientes. Si después de recurrir a todos los datos disponibles de la lengua hay distintas posibilidades de interpretación, los resultados del método comparativo y los conocimientos de tipología areal y universales lingüísticos pueden jugar un papel importante para decidir qué reconstruir. Por ejemplo, en el caso del fonema muisca representado por medio de $\left\langle\xi h_{\perp}>\right.$ en la gramática de Bernardo de Lugo y por $<\mathrm{z}>$ en otras fuentes, para decidir si se trataba de una africada alveolar sorda o sonora, resultan importantes los siguientes datos: (a) de acuerdo con el método comparativo, al menos parte de las $\left\langle\zeta_{\mathbf{H}}\right\rangle$ se derivan de $/ *$ ts/ (Constenla Umaña 1981: 241), (b) en tanto en Colombia y los territorios más cercanos de los países vecinos /ts/ es muy frecuente (existe, por ejemplo, en cogui, chimila, damana, páez, chamí, puinave, cofán, colorado, cayapa, camsá y guambiano), /dz/ se da raramente: las únicas lenguas relativamente próximas al muisca en que tengo noticia que se dé son el cofán y el quechua ecuatoriano (y, en ambos casos, forma parte de un subsistema de africadas, con oposición entre sonoras y sordas, que incluye además a /ts/, /ți / y / $/$ g /) y (c) estos hechos areales reflejan la mayor frecuencia en las lenguas de las africadas sordas (Ladefoged 1996: 90).

\section{El método restitutivo: reconstrucción de los efectos de las desviaciones de transcripción}

\subsection{Los dos tipos fundamentales de desviaciones de las transcripciones no profesionales con respecto a la fonemática de la lengua}

Gleason (1961: 272-273) caracteriza dos tipos fundamentales de desviación que una transcripción preliminar de una lengua puede presentar con respecto a la transcripción fonemática 
correcta de la misma: infradiferenciación (empleo del mismo símbolo gráfico para transcribir dos o más fonemas distintos) y supradiferenciación (empleo de dos o más símbolos gráficos distintos para un mismo fonema, generalmente por representación de fonos que alternan como realizaciones suyas). Un tercer tipo que añade, la mala segmentación, no es realmente fundamental, pues en los casos en que se trata de representación gráfica de un fonema como secuencia de fonemas implica infradiferenciación (entre un fonema y una secuencia), y en aquellos en que se trata de representación de una secuencia de fonemas por medio de un símbolo único diferente, implica supradiferenciación.

En la medida en que la supradiferenciación no se traslape con la infradiferenciación y se mantenga independiente, su existencia es poco problemática, pues la transcripción que la presente es fonéticamente adecuada y de ella se puede derivar una transcripción fonemática correcta. Lo mismo no sucede en el caso de la infradiferenciación, que implica la omisión parcial o total de distinciones significativas.

Gleason (ibídem: 273) comenta al respecto: "Los registros de personas sin experiencia en la transcripción fonética presentan generalmente dificultades mucho mayores, pues la infradiferenciación aparecerá en ellos muy probablemente. No hay proceso para separar lo pertinente de lo que no lo es en tales casos, dado que algunas distinciones significativas se omiten por completo en los datos. La infradiferenciación y los errores garrafales pueden encontrarse y eliminarse únicamente por medio de la referencia renovada al habla del informante, si bien, con frecuencia, pueden surgir, de los registros mismos, sospechas fuertes de su existencia."

Como en muchos casos no podemos ya recurrir a informantes, para aproximarnos a la estructura de las lenguas que se encuentren en dicha situación, la solución, cuando esto ocurre, es una aproximación a los datos, como la que aquí se está proponiendo, que nos permita no solo sospechar la existencia de desviaciones con respecto a una transcripción correcta, sino reconstruir esta en la medida en que ellos lo hagan posible.

\subsection{Efectos de la infradiferenciación y la supradiferenciación: las correspondencias en la restitución y los pasos fundamentales de este método}

Hay una obvia semejanza entre los dos tipos fundamentales de desviación en la transcripción y los dos cambios fonológicos fundamentales.

La infradiferenciación corresponde a la fusión, pues redunda en la supresión de diferencias fonemáticas. La supradiferenciación, que en principio lo que implica es simplemente una transcripción fonética, cuando se combina con la infradiferenciación, resulta perfectamente paralela a la escisión. Un ejemplo se da en los datos de guatuso de Lehmann (1920: 382-391, 404-415) en los que $<$ b $>$ aparece en alternancia a veces con $<\mathrm{f}>(<\mathrm{xafúuti}>$, $<$ xabúñti $>$ 'yerno') y otras con $<$ p $>(<æ p e>$, $<$ æbe $>$ 'no'), lo cual se debe a que Lehmann utilizó la $<$ b $>$ para transcribir tanto el alófono [\$] del fonema / $\$$ / como el alófono [b] del fonema /p/ (en ambos casos hay variación libre en la posición intervocálica).

Los efectos de la infradiferenciación y la supradiferenciación son, pues, paralelos a los que en la evolución fonológica producen, respectivamente, la fusión y la escisión.

Las transcripciones asistemáticas de un mismo transcriptor no son, normalmente, anárquicas. Los transcriptores interpretan los sonidos que escuchan condicionados ante todo por su 
propia lengua (y, en menor grado, por otras que conozcan) y los transcriben partiendo, en principio, de las convenciones del sistema de escritura que estén empleando. De acuerdo con mis observaciones, las desviaciones que cometen tienden a ser consistentes, de modo que, en los mismos entornos, suelen presentarse, por lo menos de manera predominante, las mismas.

Enfrentados a corpus de transcripción no profesional o de transcripción preliminar, una vez reunido el léxico total disponible y establecidas las aclaraciones grafemáticas, se procederá a encontrar todas las inconsistencias de transcripción, o sea, las variantes de transcripción que se den entre elementos glosados de la misma manera cuya forma sea sólo parcialmente diferente. Esto se podrá extender posteriormente a elementos con glosas distintas, pero relacionadas. Surgirán entonces, tanto en los datos de un mismo transcriptor como en los de distintos transcriptores, correspondencias entre distintos símbolos gráficos. Cuando estas correspondencias reaparecen, son indicio de la presencia de infradiferenciaciones o supradiferenciaciones en los datos. Las correspondencias de este tipo son precisamente el elemento que nos permite recuperar oposiciones no representadas por los transcriptores. Esta es una diferencia importante entre la situación apropiada para la restitución, la de la existencia de varias transcripciones asistemáticas individuales, y aquella a la que suelen referirse los manuales de lingüística diacrónica en su sección de uso de los testimonios escritos, la de la existencia de un alfabeto convencional, consolidado y tradicional. En este último caso, como ha señalado Hoenigswald (1960: 5)10 "si una oposición no se representa..., como regla, esto no puede descubrirse a partir de los indicios gráficos tan solo."

En suma, partiendo de una lista de elementos de los cuales dispongamos distintas transcripciones de dos o más transcriptores podremos determinar correspondencias sistemáticas. Esto se puede comprobar fácilmente por medio de casos de control, como los constituidos por aquellos en que disponemos de cuerpos de datos transcritos asistemáticamente de una lengua hablada cuya fonología se haya descrito apropiadamente. Usaré como ejemplo el caso del bribri, lengua chibchense de Costa Rica. Si observamos datos de autores como Thiel (1884) y Pittier (1898), al comparar el uso de letras que normalmente representan vocales anteriores, encontraremos que surgen numerosos ejemplos de tres conjuntos de correspondencias (i-i, e-e, e-i) como los que se dan a continuación (se coloca en una columna adicional la representación fonemática acorde con mi análisis del sistema fonológico de la lengua, Constenla Umaña 1981: 106-17):

\section{CONSTENLA}

$\mathrm{i}-\mathrm{i}$

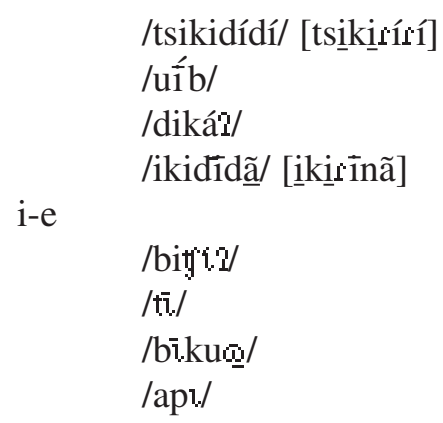

\section{THIEL}

\begin{tabular}{|c|c|}
\hline tsquiríri & tskiri-ri \\
\hline huíb & uíb \\
\hline dicá & di-ká \\
\hline quirina & kirína \\
\hline biché & $\mathrm{bi}+\mathrm{t}$ \\
\hline te & $\mathrm{ti}$ \\
\hline be-cvo & bík-uo \\
\hline pe & apiź \\
\hline
\end{tabular}

'amarillo' 'mono congo' 'espina' 'se enfermó'

'alacrán' 'labranza' 'estrella' 'sangre' 
e-e

\begin{tabular}{|c|c|c|c|}
\hline /tabE/ [tabs] & tabé & tebé & 'cuchillo, hierro' \\
\hline 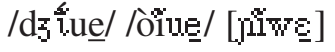 & nigve & ñí-ue & 'de día' \\
\hline$/ \mathrm{w} \mathrm{b} /[\mathrm{w} \xi \mathrm{b} w \bar{\theta} \mathrm{m}]$ & vem & UËn & 'rana arborícola' \\
\hline 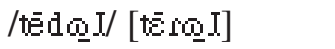 & terl & ter-ul & 'seis' \\
\hline
\end{tabular}

La correspondencia e-i es resultado de la infradiferenciación de $/ \mathrm{l} /$ con $/ \varepsilon /$ en el caso de Thiel y con /i/ en el caso de Pittier.

La consistencia de las transcripciones asistemáticas, sin embargo, no es normalmente absoluta, sino que es una tendencia fundamentalmente estadística, de modo que aunque un resultado predomine, pueden darse otros. En consecuencia, en los datos de cualquiera de los dos autores mencionados, encontraremos una minoría de casos en que, en los mismos entornos de los ejemplos citados, Thiel transcribe $/ . /$ por medio de i y Pittier por medio de e. A veces, encontramos en un mismo autor transcripciones alternativas de los mismos elementos. Ejemplos de estas dos circunstancias son:

\section{CONSTENLA THIEL PITTIER}

$\begin{array}{lll}\text { /buịil bohíh } & \text { bú-e } & \text { 'tiquisque' (Xanthosoma violaceum) } \\ \text { /suIúl./ } & \text { surue, suru-e, } & \text { surú-i 'muy malo' }\end{array}$

El número en que se presentan transcripciones alternativas varía mucho según el autor. Por ejemplo, en Thiel y Pittier son más bien escasas (3,5\% y 6,25\%, respectivamente, de la muestra observada); en cambio, en Arroyo (1951) tienen una frecuencia bastante notable (el $19,6 \%$ de la muestra).

Debido a las transcripciones alternativas, se producen traslapes entre correspondencias. Tal es el caso de la correspondencia Thiel $<$ e $>$ Pittier $<\mathrm{i}>$, que se traslapa con la correspondencia Thiel $<$ e $>$ Pittier $<$ e $>$ a partir del ejemplo dado anteriormente de vacilación en la transcripción de Pittier. Cuando se produce un traslape de correspondencias en un mismo rubro léxico, se considera que la representada en ese caso es la que, no siendo caso único, no esté constituida por la misma letra en todas las fuentes de datos (esto como prevención de la infradiferenciación). Si hay varias correspondencias que cumplen este requisito, se escogerá la que sea más frecuente en el entorno en cuestión.

El primer paso del método restitutivo como tal, una vez efectuados los preliminares grafemáticos, es el establecer un inventario lo más exhaustivo posible de correspondencias.

El segundo es tabular la distribución de cada correspondencia de acuerdo con los entornos.

El tercero consiste en determinar entre cuáles correspondencias se da una mayor semejanza, tanto por la naturaleza en sí de los sonidos presuntamente representados por las letras que las integran, como por la relación de las letras con cada fuente. Así pues, una correspondencia $<$ e-e-i $>$ es más semejante a una $<$ i-e-i $>$ que a una $<\mathrm{i}-\mathrm{i}$-e $>$.

Tras estos pasos iniciales, se procede a comparar la distribución de correspondencias con semejanza máxima, comenzando por aquellas que se haya observado alternar en los mismos elementos. Como los sonidos en un análisis fonemático, la distribución de las correspondencias 
puede ser (a) potencialmente opositiva (si se dan en los mismos entornos) o (b) complementaria (si se dan en entornos distintos).

Conviene iniciar estas comparaciones por las correspondencias con menor frecuencia de aparición y limitadas a posiciones que no sean la intervocálica.

\subsection{La distribución complementaria entre correspondencias: restitución de una situación de supradiferenciación y de un único fonema}

La distribución complementaria entre correspondencias es la situación más simple que puede darse y de inmediato lleva a la reconstrucción de una situación de supradiferenciación y de un único fonema. Tal es el caso, en muisca, de la correspondencia entre la secuencia $<u y\rangle$ (transcripción de Bernardo de Lugo) y $<u y>$ (transcripción de otras fuentes) que se presenta sólo precedida por consonante labial y la correspondencia $<\bar{Y}>$ (transcripción de Bernardo de Lugo) y $<y>$ (transcripción de otras fuentes) que es la que se presenta en los demás entornos. Otro ejemplo, en este caso del andaquí, es el de la correspondencia $<\mathrm{d}-\mathrm{d}>$, que aparece solamente tras vocal nasal (representada como vocal seguida por $<\mathrm{n}>$ ) frente a la correspondencia $<\mathrm{t}-\mathrm{t}\rangle$, que se presenta en los demás entornos (Coronas Urzúa 1993: 27-8).

La sola restricción de la aparición de una letra a un entorno que no sea el más universal de los posibles en las lenguas, la posición intervocálica, ya es indicio de distribución complementaria del sonido que representa con alguno otro. Este indicio es seguro si la letra representa un sonido para cuyo reconocimiento el transcriptor estaba capacitado a partir de las lenguas bien conocidas por él y el sistema de escritura empleado.

\subsection{La distribución potencialmente opositiva: aparición en entornos idénticos}

Esta pauta de distribución es, en cambio, más problemática en el caso que nos ocupa, porque puede reflejar tanto una situación de supradiferenciación como una de infradiferenciación y carecemos de los informantes que, en el caso de una lengua viva, podrían sacarnos rápidamente de la duda.

Para intentar determinar cuál es la situación real, un factor fundamental que debe tomarse en cuenta es la capacidad de los transcriptores y del alfabeto empleado por ellos para representar apropiadamente diferencias entre sonidos. Según las lenguas que conozcan, entre las cuales la más importante para efectos de percepción es la materna, los transcriptores estarán capacitados o no para diferenciar determinada pareja de sonidos. Esta capacidad dependerá sobre todo de si, por lo menos, en alguna de las lenguas previamente conocidas existe oposición entre los sonidos que componen la pareja (es decir, si pertenecen a distintos fonemas).

Las lenguas de las cuales se parte determinan también con qué se infradiferencia. Por ejemplo, Olavarrieta (hacia 1788, incluido en Fernández Guardia y Fernández Ferraz 1892),

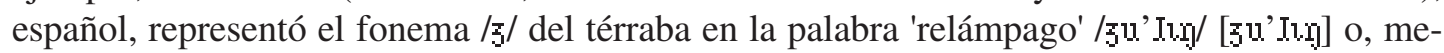
nos frecuentemente, [ $\left.\left.5 \mathrm{Fu}^{\mathrm{i}} \mathrm{InTy}\right]\right)$ por medio de $<\mathrm{y}>(<\mathrm{yuren}>$ ) en tanto que Thiel (1884), alemán, usa el trígrafo $<\mathrm{sch}>(<$ schigering $>)$. El sonido más semejante a $\left[\begin{array}{l}\boldsymbol{3} \\ ]\end{array}\right.$ presente en el español (tanto costarricense como peninsular) es [j], en tanto que en alemán es [j]. Como consecuencia, al transcribir el térraba, lengua en que se oponen $/ 5 /, /\lceil/$ y [j] (según mi análisis, alófono de [i] en 
posición inicial de sílaba), en el caso de Olavarrieta se produce infradiferenciación de $/ 5 /$ con [j] (transcribe, por ejemplo, $\gamma^{\prime} \mathrm{i} \omega \mathrm{k} /$ 'fuego' como <yuc> ) y, en el de Thiel, de $/ \xi /$ con $/ j /$ (transcribe /' ¡i/ 'nosotros, inclusivo' como <schi >).

Por otra parte, si el sistema de escritura empleado no tiene una representación adecuada y consistente de una oposición, esta puede no ser representada por el transcriptor, aunque se dé en su lengua materna. Por ejemplo, Gabb (1875) no representó en sus transcripciones del bribri, hechas. en el caso de las vocales con ortografía española, la oposición de $/ \mathrm{v} / \mathrm{a} / \varepsilon / \mathrm{y}$ a /i/, a pesar de que se da en su lengua materna, el inglés.

\subsubsection{La restitución de una situación de supradiferenciación y de un solo fonema: variación libre}

Cuando dos o más correspondencias con un grado máximo de semejanza se presentan en los mismos entornos, es recomendable probar, en primer lugar, la hipótesis de que se trata de una situación de supradiferenciación y, en consecuencia, que lo que se da en la lengua es variación libre entre fonos pertenecientes a un mismo fonema.

Los siguientes son indicios favorables para esta hipótesis:

2.4.1.1. Las correspondencias alternan en rubros con el mismo significado. Por ejemplo, en los datos de jirajara la correspondencia $<\mathrm{s}>$ (Oramas) $-<\mathrm{s}>$ (Jahn) alterna con la correspondencia $<\mathrm{s}>$ (Oramas) - <sh $>$ (Jahn), como en 'fruto del cardón' <ispó $>$ (Oramas, Jahn), <ishpó $>$ (Jahn).

Con frecuencia la variación libre se combina con la distribución complementaria, de modo que está restringida a determinado(s) entorno(s). La alternancia de dos o más correspondencias restringida a determinada posición o determinadas posiciones, en tanto en los otros entornos sólo se encuentra una de las correspondencias en cuestión, indica que se da esta combinación de pautas de distribución.

2.4.1.2. Las letras incluidas en correspondencias que alternan en elementos con idéntico significado representan sonidos contiguos en el espacio fonológico. Si esto se da con seguridad (para determinar lo cual hay que tomar en cuenta tanto las lenguas conocidas por el transcriptor como el alfabeto empleado) no cabe la infradiferenciación, de modo que la alternancia necesariamente se deberá a variación libre. Para ejemplificar de manera bien obvia, en el lenca de Chilanga, se encuentran las siguientes correspondencias en el mismo entorno (intervocálico): $<\mathrm{s}>$ (Lehmann) - <s $>$ (Peccorini), <z> (Lehmann) - <s $>$ (Peccorini). Lehmann, además de estar capacitado por su lengua materna (el alemán) para distinguir, en posición intervocálica, la diferencia fonética entre una fricativa alveolar sorda y una sonora, empleaba el Alfabeto General de Lepsius en el que el primer sonido se representa por medio de $<$ s $>$ y el segundo por medio de $\langle\mathrm{z}\rangle$. En consecuencia, no cabe la menor posibilidad de que Lehmann pudiera haber confundido un sonido intermedio en el espacio fonológico a veces con [s] y a veces con $[\mathrm{z}]$ y hubiera, así, incurrido en infradiferenciación.

2.4.1.3. Se dan, en número semejante y sin relación especial con ningún entorno, correspondencias inversas, como podrían ser, transcriptor $1<\mathrm{i}>$ - transcriptor $2<\mathrm{e}>$, transcriptor $1<\mathrm{e}>$ - transcriptor $2<\mathrm{i}>$. Tal es el caso del andaquí, en el que se presenta constantemente este fenómeno: 


\section{Fuente anónima del siglo XVIII}

dorada
machete
agua
pejerraya
sí
tapir

\author{
bicahi \\ binixi \\ jejê \\ jejohé \\ ynszi \\ szuntijohé
}

M.M. Albis, siglo XIX
vegaé
benije
jiji
jijoe
ince
condefui

Aunque no cabe esperar una consistencia absoluta en la transcripción con infradiferenciación, mis observaciones me llevan a pensar que el mismo transcriptor tiende a interpretar, con pocas excepciones, de la misma manera el mismo sonido en un mismo entorno: por ejemplo, el fonema /./ del bribri fue interpretado como $<\mathrm{e}>$ por Thiel de manera consistente excepto en los casos en que lo preceden los sonidos [\$] $]$, [I] y [w]

2.4.1.4. Entre tres correspondencias fonéticamente semejantes que, al menos en algunos casos, se encuentran en alternancia en rubros con idéntico significado, la frecuencia de las que incluyen distintos sonidos, que denominaré heterogéneas, es notablemente superior a la de aquellas que presentan el mismo sonido en todos los transcriptores, que denominaré homogéneas. Cuanto mayor sea la diferencia de frecuencia a favor de las correspondencias heterogéneas, más factible es que la relación entre los sonidos sea de variación libre.

2.4.1.5. Los sonidos que alternan en las correspondencias aparecen intercambiados en préstamos tomados de otras lenguas en las que pertenecen a distintos fonemas. En jirajara (Campos Bolaños 1994: 52), [s], [j] y [t j]'] alternan en transcripciones de las mismas palabras. Un ejemplo, en el caso de [s] y [t]'], es 'cabestro', transcrito tanto <tchikú> como < sicú>. Que se trata de variación libre se confirma de manera definitiva al observar préstamos de origen castellano como: <lesí> 'leche', <maset> 'machete' y <chamor samor> 'zamuro'.

2.4.1.6. Existen declaraciones de observadores que explícitamente señalan la existencia de la variación libre. Por ejemplo, Porras Ledesma (1959: 159) señala claramente respecto de la alternancia entre [B] y [\$] en guatuso: "Una misma palabra, abójora, por ejemplo, se pronuncia afójora minutos más tarde, por la persona que antes la había citado; igual sucede con todos los demás informantes."

\subsubsection{La restitución de una situación de infradiferenciación y de una oposición fonemática no obvia}

Esta sección se dedica fundamentalmente a los casos de correspondencias heterogéneas que incluyen sonidos a su vez presentes en correspondencias homogéneas que coexisten en los mismos entornos con ellas.

De acuerdo con lo señalado anteriormente, la falta de contigüidad en el espacio fonológico de los sonidos representados por las letras incluidas en correspondencias fonéticamente semejantes que se dan en los mismos entornos, es un requisito para que se pueda plantear la posibilidad de infradiferenciación. 
Las situaciones tratadas en los apartes siguientes constituyen indicios de infradiferenciación y de existencia de oposiciones ocultadas por este fenómeno.

2.4.2.1. Las correspondencias comparadas se dan en los mismos entornos, pero no se observan en alternancia en los mismos morfemas.

2.4.2.2. Dos correspondencias homogéneas (por ejemplo, a-a y b-b) alternan con la heterogénea relacionada $(\mathrm{a}-\mathrm{b})$, pero no alternan entre sí.

2.4.2.3. Las correspondencias comparadas con base en la semejanza fonética no incluyen casos de inversión, o, en caso de incluirlos, una de las correspondencias inversas es poco frecuente y está limitada a entornos muy específicos.

2.4.2.4. Las correspondencias diferencian elementos que, según algunas transcripciones, serían homófonos. Por ejemplo, en la transcripción de Pittier las palabras bribris 'quién' y 'padre' son homófonas: se transcriben como <iž̃i > ambas. Pero, para Arroyo, 'quién' es <yí> y 'padre' $<$ yê $>$. La correspondencia presente en el caso de 'padre' es $<\mathrm{i}$-e $>$, mientras, en el caso de 'quién' es $<\mathrm{i}-\mathrm{i}>$.

2.4.2.5. En un mismo entorno, se presentan con frecuencia semejante una correspondencia heterogénea (tipo a-b), con inversión (b-a) o sin ella, y las correspondencias homogéneas relacionadas (a-a, b-b).

2.4.2.6. Los sonidos que integran una correspondencia heterogénea se mantienen inalterados en las palabras tomadas en préstamo a lenguas en las que pertenecen a distintos fonemas. Por lo que respecta a la antigüedad de la oposición en la lengua, cuando el número de préstamos es pequeño, lo más probable es que existiera desde antes de esta influencia. Cuando hay una presencia masiva de préstamos, podría suceder que la oposición no existiera originalmente en la lengua y que hubiera surgido gracias a los préstamos.

\subsection{Restitución de oposiciones obvias por no darse infradiferenciación o por haber- se documentado explícitamente}

Existe oposición obvia cuando en los mismos entornos coexisten correspondencias homogéneas que no alternan nunca en transcripciones de un mismo morfema.

En algunos casos, hay, además, declaraciones explícitas de observadores sobre la existencia de la oposición, como la siguiente, incluida en el Diccionario y gramática chibcha, obra anónima del siglo XVII (González de Pérez 1987: 72), que incluso aporta parejas mínimas: "...estas sílabas ha, he, hi, ho, hu, hy, por las quales se distinguen unus vocablos de otros, como se ue en los siguientes: vaya significa madre y vahaya, el difunto; otro: chica, el suegro del hierno y chihica, la carne." 


\subsection{Irrecuperabilidad de oposiciones}

El método de restitución, como cualquier otro, no puede ir más lejos de lo que permiten los materiales disponibles. En consecuencia, en muchos casos habrá habido en la lengua oposiciones que no podemos recuperar. Esto ocurre, sin duda, con especial frecuencia en el caso de los suprasegmentales, pero no deja, de ningún modo, de darse en el caso de los segmentales. La restitución nunca puede pretender una recuperación total de la estructura fonológica de la lengua. Pero, de hecho, para todo efecto práctico, una lengua es lo que conocemos de ella. Como lo señaló Bloch en sus Postulados para la lingüística descriptiva (Fought 1967: 6) 'los elementos que el analista no puede discriminar son lo mismo'.

A veces, incluso, podemos llegar a la conclusión de que determinado tipo de rasgo existió en la lengua, pero no lo podemos incorporar a la transcripción fonemática porque fuera de algún ejemplo en particular, no conocemos su desempeño en la inmensa mayoría de los morfemas (o las palabras). En este caso, el dato será plenamente aprovechable, por ejemplo, para estudios de carácter areal, pero de utilidad muy limitada para la aplicación del método comparativo.

\subsection{Síntesis de los pasos de la restitución}

De acuerdo con la exposición previa, los pasos del método son los siguientes:

a. Todos los materiales disponibles de la lengua se reúnen y se elabora un léxico con las entradas en la lengua en que se realiza el estudio y sus equivalentes en la lengua objeto de la restitución. En este léxico se agruparán en una misma entrada todas las versiones de un mismo lexema de la lengua objeto. Si el elemento presenta distintas acepciones, se escoge una como lema de la entrada.

b. Se efectúa un estudio grafemático de los datos de cada fuente con el fin de determinar los valores fonéticos de las letras empleadas. Con base en este análisis se plantean las equivalencias entre los alfabetos de las distintas fuentes.

c. Se procede a establecer un inventario de las correspondencias que se repitan. Se tabula la distribución de cada correspondencia según los entornos en que se presentan.

d. Se establecen parejas de correspondencias con una relación máxima. El grado máximo de relación se determina a partir de la alternancia de las correspondencias en los mismos elementos y su semejanza fonética.

e. Se determina si existe oposición o no entre las correspondencias. Las correspondencias entre las que se comprueba oposición se asignan a distintos fonemas. Las correspondencias entre las que se comprueba relaciones de variación libre o distribución complementaria se asignan a un mismo fonema. Esta etapa del procedimiento producirá un inventario de fonemas.

A partir de los resultados obtenidos hasta el momento, se dan los siguientes pasos adicionales: 
e. Se redactan los resultados a partir del inventario fonemático obtenido, describiendo las realizaciones de cada fonema y señalando cuál es la representación de estas en cada transcriptor.

f. Se produce una transcripción fonemática de cada lexema del léxico general organizado inicialmente. A partir de este corpus transcrito fonemáticamente, se obtienen conclusiones sobre otros aspectos de la fonología de la lengua como la distribución y frecuencia (de diccionario) de los fonemas.

g. La presentación final estará integrada por el estudio grafemático y la descripción de la fonología de la lengua.

\section{8. ¿Se puede restituir con base en una única fuente de datos?}

Para aplicar el método restitutivo, la existencia de dos o más transcriptores resulta indispensable. Cuando tenemos datos de un único transcriptor, podemos realizar un estudio grafemático, con base en el cual se podrán eliminar ciertos efectos de la supradiferenciación, como los casos obvios de distribución complementaria y aquellos de variación libre que hayan sido percibidos como tales por el transcriptor (véase 2.4.1.5.). En este tipo de situación, los casos de variación libre que hayan pasado desapercibidos para el transcriptor y las alternancias resultantes de la infradiferenciación resultan, lamentablemente, prácticamente indistinguibles, y es poco probable que se pueda ir más allá del diagnóstico de la condición defectuosa de los datos.

\section{Carácter fundamentalmente fonológico y descriptivo de la restitución}

Como el método comparativo de la lingüística diacrónica, la restitución es fundamentalmente fonológica. En el método comparativo, una vez se han reconstruido los protofonemas, se pueden reconstruir, si los datos se prestan para ello, distintas entidades sintácticas de la protolengua como palabras y frases. Las conclusiones que obtengamos sobre la gramática de la protolengua serán el resultado del análisis de estas estructuras, que en principio no tiene nada de distinto al que podamos hacer de datos semejantes de cualquier lengua. En realidad, una vez establecidas las correspondencias entre los fonemas de las lenguas comparadas, como lo demostró Hoenigswald (1950: 357), el procedimiento seguido no difiere en lo esencial de cualquier análisis sincrónico:

La reconstrucción por medio del método comparativo ... es esencialmente un problema de fonemática en el cual el lugar de los alófonos es asumido por conjuntos de correspondencias fonéticas que son semejantes fonéticamente (comparten un componente) y están en distribución complementaria.

Lo mismo sucede en la restitución. Cada correspondencia juega el papel de un sonido distinto que debemos determinar si se opone o no a los sonidos (correspondencias) más estrechamente relacionados por la semejanza fonética. Una vez determinamos los fonemas de la lengua a partir de las correspondencias, podemos transcribir fonemáticamente las palabras, 
frases y oraciones de que dispongamos y analizarlas gramaticalmente para describir este otro nivel de la lengua. También podemos hacer un diccionario con los lexemas transcritos fonemáticamente.

A pesar de esta semejanza con el método comparativo y de que las entidades lingüísticas que se recuperan parcialmente por su medio pertenezcan al pasado -son lenguas extintas o estados previos de lenguas que todavía se hablan-, la restitución es un método fundamentalmente descriptivo y sincrónico, no diacrónico, ya que la reconstrucción que lleva a cabo no nos remonta de un estado de lengua a otro anterior.

\section{Notas}

1. Una versión abreviada de este trabajo se presentó como ponencia de plenaria en el XIII Congreso de la Sociedad Chilena de Lingüística, celebrado en la Universidad de La Serena, del 11 al 15 de octubre de 1999.

2. En el desarrollo de este trabajo, al citar obras escritas en inglés, daré mi traducción del pasaje en cuestión, que se incluirá en el idioma original como nota.

3. Before any written material can be used for linguistic purposes, philological screening has to establish its fit to the real language, as well as its fit to the real cultural situation, if semantic material is needed.

4. The analysis, interpretation and restauration of archaic texts is termed philology. Solid philological investigation is the foundation of most historical linguistic research.

5. The analysis of the philologist establishes how faithfull a given text is in its representation of language.

6. The following languages are thought to be extinct... These need much more work.... Philological studies of all available information are needed, including the study of toponyms, onomastics, extant vocabularies, grammars, texts, etc.

7. The most important need for Lencan is a thorough philological investigation of both languages, especially H.L.

8. El nombre restitución no ha sido usado anteriormente, hasta donde tengo conocimiento, de manera sistemática para denominar ningún método lingüístico. No obstante, después de haberlo adoptado para el método que expongo en este trabajo, me he dado cuenta de que Meillet (1937: 39) lo usó, por lo menos una vez, para referirse a la reconstrucción comparativa.

9. Las letras usadas por los transcriptores y las representaciones de palabras por su medio se enmarcan entre $<>$.

10. If a contrast is not represented ..., this cannot as a rule be discovered through graphic evidence alone.

\section{Bibliografía}

Albis, Manuel María. 1860-1. "The Indians of Andaqui, New Granada. Notes of a traveler. Translated by J.S. Thrasher". Bulletin of the American Ethnological Society (Nueva York). 1: 53-72. 
Anónimo del siglo XVIII. 1928. "Vocabulario Andaquí-Español". En: Lenguas de América volumen I. Catálogo de la Real Biblioteca, tomo VI: Manuscritos, 175-195. Madrid.

Anttila, Raimo. 1972. An Introduction to Historical and Comparative Linguistics. MacMillan Publishing Co., Inc., Collier MacMillan Publishers. Nueva York, Londres.

Arrieta Espinoza, Anita. 1992. "Tipología fonológica y morfosintáctica del timote". Tesis de maestría. Universidad de Costa Rica.

Arroyo Soto, Víctor Manuel. 1951. "Lenguas indígenas costarricenses". Tesis de licenciatura. Universidad de Cista Rica.

Bertoglia Richards, Mafalda. 1988. "La lengua cacaopera: fonología y léxico". Tesis de maestría. Universidad de Costa Rica.

Campbell, Lyle. 1979. "Middle American Languages". En: Campbell, Lyle y Marianne Mithun (compiladores). The languages of Native America: Historical and Comparative Assessment, 902-1000. University of Texas Press. Austin.

Campos Bolaños, Gerardo. 1994. "La lengua jirajara. Análisis lingüístico de los materiales existentes". Tesis de licenciatura. Universidad de Costa Rica.

Cerrón Palomino, Rodolfo.1995. La lengua de Naimlap (Reconstrucción y obsolescencia del mochica). Fondo Editorial de la Pontificia Universidad Católica del Perú. Lima.

Constenla Umaña, Adolfo. 1981. "Comparative Chibchan Phonology". Tesis doctoral. Universidad de Pensilvania.

1984. "Los fonemas del muisca". Estudios de Lingüística Chibcha (Universidad de Costa Rica) 3: 65-112.

Coronas Urzúa, Gabriela.1993. "La lengua andaquí: fonología y léxico". Tesis de maestría. Universidad de Costa Rica.

Fernández Guardia, Ricardo y Juan Fernández Ferraz. 1892. Lenguas indígenas de Centro América en el siglo XVIII según copia del Archivo de Indias hecha por el licenciado don León Fernández. Tipografía Nacional. San José.

Fought, John G.1967. "Chorti (Mayan): Phonology, Morphophonemicas, and Morphology." Tesis doctoral. Universidad de Yale.

Gabb, William. 1875. "On the Indian tribes and languages of Costa Rica." Proceedings of the American Philosophical Society. 14: 483-602. 
Gelb, I.J. 1952. A Study of Writing. The University of Chicago Press. Chicago.

Gleason, Henry A. 1961. An Introduction to Descriptive Linguistics. Holt, Rinehart and Winston. Nueva York.

González de Pérez, María Stella.1987. "Diccionario y gramática chibcha." Manuscrito anónimo de la Biblioteca Nacional de Colombia. Transcripción y estudio histórico-analítico. Instituto Caro y Cuervo. Bogotá.

Hoenigswald, Henry M. 1950. "The principal step in comparative grammar." Language 26: 357-64.

1960. Language Change and Linguistic Reconstruction. The University of Chicago Press. Chicago.

Jahn, Alfredo. 1973. Los aborígenes del Occidente de Venezuela. Monte Ávila Editores, C.A. Caracas.

Jeffers, Robert J. e Ilse Lehiste.1979. Principles and Methods for Historical Linguistics. The M.I.T. Press. Cambridge, Massachusetts.

Ladefoged, Peter. 1996. The sounds of the World's Languages. Blackwell Publishers. Cambridge, Massachusetts.

Lehmann, Walter. 1920. Zentral-Amerika . Primera Parte. Die Sprachen Zentral- Amerikas. 2 tomos. Verlag Dietrich Reimer. Berlín.

Lepsius, R. 1855. Das allgemeine linguistische Alphabet. Verlag von Wilhelm Hertz. Berlín.

Lugo, Bernardo de. 1619. Gramatica en la lengva general del Nuevo Reyno, llamada mosca. Barnardino de Guzmán. Madrid.

Mattéi-Muller, Marie-Claude y Paul Henley.1990. Los tamanaku: su lengua, su vida. Universidad Católica del Táchira. Caracas.

Najlis, Elena Lidia. 1966. Lengua abipona. Tomo I. Centro de Estudios Lingüísticos, Universidad de Buenos Aires.

Oramas, Luis R. 1916. Materiales para el estudio de los dialectos ayamán, gayón, jirajara, ajagua. Litografía del Comercio. Caracas.

Pittier, Henri.1898. Die Sprache der Bribri-Indianer in Costa Rica. Kaiserliche Akademie der Wissenschaften. Viena. 
Porras Ledesma, Álvaro. 1959. "El idioma guatuso (Fonética y Lexicología)". Tesis de Licenciatura. Universidad de Costa Rica.

Quirós Rodríguez, Santiago. 1984. "La lengua chorotega o mangue: análisis lingüístico de los materiales existentes". Tesis de licenciatura. Universidad de Costa Rica.

Río Urrutia, Ximena del. 1985. "El lenca de Chilanga: fonología y léxico". Tesis de licenciatura. Universidad de Costa Rica.

Rosales, Carlos J. 1919. Gramática del idioma cachiquel, escrita en 1748 por un religioso franciscano, el R.P. Fr. Carlos J. Rosales. Tipografía "San Antonio". Guatemala.

Rosenblat, Ángel. 1936. "Los otomacos y taparitas de los llanos de Venezuela. Estudio etnográfico y lingüístico". Tierra Firme (Madrid) 2: 131-53, 259-304, 439-514.

Thiel, Bernardo Augusto. 1884. Apuntes lexicográficos de las lenguas y dialectos de los indios de Costa Rica. Imprenta Nacional. San José. 